\title{
Secure Beamforming for Max-Min SINR in Multi-Cell SWIPT Systems
}

\author{
Ali A. Nasir*, Duy T. Ngo ${ }^{\dagger}$, Hoang Duong Tuan ${ }^{\ddagger}$, Salman Durrani ${ }^{\S}$, and Dong In Kim \\ * School of Electrical Engineering and Computer Science, National University of Sciences and Technology, Islamabad, Pakistan. \\ $\dagger$ School of Electrical Engineering and Computer Science, The University of Newcastle, Australia \\ $\ddagger$ Faculty of Engineering and Information Technology, University of Technology Sydney, Australia. \\ ${ }^{3}$ Research School of Engineering, The Australian National University, Canberra, Australia \\ T School of Information and Communication Engineering, Sungkyunkwan University, South Korea
}

\begin{abstract}
We consider the downlink of a dense multicell network where each cell region is divided into two zones. The users nearby their serving base station (BS) in the inner zone implement simultaneous wireless information and power transfer (SWIPT), thus harvest energy and decode information using the power splitting approach. Further, they try to eavesdrop the information intended for other users within the same cell. The users in the outer zone of each cell only implement information decoding. Our objective is to maximize the minimum user equipment (UE) signal-to-interference-and-noise ratio (SINR) under constraints on the BS transmit power, minimum energy harvesting levels of near-by users, and maximum SINR of eavesdroppers in the presence of multi-cell interference. For such a highly non-convex problem, semidefinite relaxation (SDR) may even fail to locate a feasible solution. We propose two methods to address such a difficult problem. In the spectral optimization, we express the rank-one constraints as a single reverse convex nonsmooth constraint and incorporate it into the optimization objective. In the difference-of-convex-functions iteration method, we directly solve for the beamforming vectors via quadratic programming $(\mathrm{QP})$, avoiding the matrix rank constraints. In each iteration of the proposed algorithms, we only solve one simple convex semidefinite program (SDP) or QP. Our simulation results confirm that the proposed algorithms converge quickly after a few iterations. More importantly, our algorithms yield the performance that is very close to the theoretical bound given by SDP relaxation with comparable computational complexity.
\end{abstract}

\section{INTRODUCTION}

Physical layer security is critical for simultaneous wireless information and power transfer (SWIPT). Although high received power levels help achieve desired energy harvesting requirements, they may make the wireless information more vulnerable to eavesdroppers. Thus, physical layer security technology should be included as part of SWIPT systems to guarantee secure communication [1]. Recently, secure beamforming in multiple-input-single-output (MISO) broadcast channels for optimal beamforming design under energy harvesting $(\mathrm{EH})$ and secure communication constraints has received great attention [1]-[7]. Particularly, different objectives have been studied, e.g., maximization of harvested energy among energy receivers (ERs) [2], [3], [5], minimization of the total network power in a cellular network [4], maximization

The work of S. Durrani was supported by the Australian Research Council's Discovery Project Funding Scheme (project number DP140101133). The work of D. I. Kim was supported by the National Research Foundation of Korea (NRF) grant funded by the Korean government (MSIP) (2014R1A5A1011478) of the achievable secrecy rate [5], [6], and minimization of transmit power in single-cell networks [7].

In order to design optimal beamforming vectors, [1]-[5] recast the formulate nonconvex problem as an equivalent semidefinite program (SDP) with rank-one matrix constraints. The typical approach in these works is to ignore non-convex rank-one constraints and solve for resulting semidefinite relaxation (SDR) problem. However, it is known that the rank-one constraint relaxation may not be tight and solutions of rank greater than one may result [6], [7]. In such cases, the optimal solution achieved by SDR can only serve as a performance bound [7].

To deal with rank-one matrix constraints, [8] proposes the randomization technique. However, as shown in our present paper and [9], the performance of such a method is far from optimal performance. Recently, [6] recommends the use of rank-reduction procedure of [10] to recover the rank-one solution. However, the rank reduction technique of [10] while giving a lower rank solution may not be able to locate a rank-one solution in general. With the obtained lower-rank solution, one still needs to employ further techniques such as randomization to generate a rank-one solution. The work of [7] reveals a sufficient condition for achieving rank-one solutions for the relaxed problem.

In this paper, we consider a dense multicell network where the multi-antenna serving BS in each cell communicates with multiple users. Each cell region is divided into two zones. The users nearby their serving BS in the inner zone implement SWIPT, thus harvest energy and decode information using the power splitting (PS) approach [11], [12]. Further, they try to eavesdrop the information targeted for other users in the respective cells. The users in the outer zone of each cell only implement information decoding. Our design objective is to maximize the minimum user equipment (UE) signalto-interference-and-noise ratio (SINR) under constraints on the BS transmit power, minimum energy harvesting levels of near-by users, and maximum SINR of eavesdroppers in the presence of multi-cell interference. We observe through extensive simulations that solving an SDR alone does not return a rank-one solution for almost $88.5 \%$ of the cases considered. Different from existing approaches, we propose optimization methods that directly find the rank-one optimal solution without assuming any conditions. We propose two methods to efficiently and optimally solve rank-constrained 
problems. In the spectral optimization method, we express the rank-one constraints as a single reverse convex nonsmooth constraint and incorporate it in the optimization objective. In the difference-of-convex-functions iteration method, we directly solve for the beamforming vectors via quadratic programming $(\mathrm{QP})$, bypassing the matrix rank constraints and matrix optimization. Each iteration of the proposed algorithms only involves one simple convex SDP or QP. Numerical results confirm that our algorithms converge quickly after very few iterations. Significantly, the performance of our algorithms is very close to the theoretical bound given by SDP relaxation with comparable computational complexity.

\section{System Model and Problem Formulation}

We consider the downlink of a dense network consisting of $K$ small cells with universal frequency reuse. The BS of a cell $k \in \mathcal{K} \triangleq\{1, \ldots, K\}$ is equipped with $M>1$ antennas and it serves $N_{k}$ single-antenna UEs within its cell. By BS $k$ and $\mathrm{UE}(k, n)$, we mean the BS that serves cell $k$ and the UE $n \in \mathcal{N}_{k} \triangleq\left\{1, \ldots, N_{k}\right\}$ of the same cell, respectively. The $N_{k}$ UEs in cell $k$ are divided into two groups, i.e., i) $N_{1, k}$ zone-1 users, which are located nearby their serving BS inside the inner circle, and ii) $N_{2, k}$ zone-2 users, which are located far from their serving BS in the outer circle. We have $N_{k}=N_{1, k}+N_{2, k}$. By UE $\left(k, n_{1}\right)$ and $\operatorname{UE}\left(k, n_{2}\right)$, we mean UE $n_{1} \in \mathcal{N}_{1, k} \triangleq\left\{1, \ldots, N_{1, k}\right\}$ in zone-1 and UE $n_{2} \in$ $\mathcal{N}_{2, k} \triangleq\left\{N_{1, k}+1, \ldots, N_{k}\right\}$ in zone-2 of cell $k$, respecitively.

Let us denote $\mathbf{w}_{k, n} \in \mathbb{C}^{M \times 1}$ as the beamforming vector by BS $k$ for its UE $(k, n)$. Let $\mathbf{h}_{\bar{k}, k, n} \in \mathbb{C}^{M \times 1}$ be the flat fading channel vector between $\mathrm{BS} k$ and $\mathrm{UE}(k, n)$, which includes the effects of large-scale pathloss and small-scale fading, where $\bar{k} \in \mathcal{K}$. We denote $\mathbf{v}_{k}$ as the artificial noise vector added by BS $k$ to deal with eavesdropping [1].

We assume that zone-1 users, being close to their serving $\mathrm{BS}$, implement SWIPT, such that UE $\left(k, n_{1}\right)$ applies the power splitting (PS) technique to coordinate both information decoding (ID) and energy harvesting (EH). The power splitter divides the received signal into two parts in the proportion of $\alpha_{k, n_{1}}: 1-\alpha_{k, n_{1}}$, where $\alpha_{k, n_{1}} \in(0,1)$ is termed as the PS ratio for $\mathrm{UE}\left(k, n_{1}\right)$. The $\alpha_{k, n_{1}}$ portion of the received signal power is used for ID while the remaining $1-\alpha_{k, n_{1}}$ portion of the received signal power is used for EH [11].

The received SINR of UE $(k, n)$ is given by

$$
\operatorname{SINR}-U E_{k, n}=f_{k, n}(\mathbf{w}, \mathbf{v}, \boldsymbol{\alpha}) \triangleq \frac{\left|\mathbf{h}_{k, k, n}^{H} \mathbf{w}_{k, n}\right|^{2}}{\varphi_{k, n}(\mathbf{w}, \mathbf{v}, \boldsymbol{\alpha})}
$$

where we denote $\mathbf{w} \triangleq\left[\mathbf{w}_{k, n}\right]_{k \in \mathcal{K}, n \in \mathcal{N}_{k}}, \mathbf{v} \triangleq\left[\mathbf{v}_{k}\right]_{k \in \mathcal{K}}, \boldsymbol{\alpha} \triangleq$ $\left[\alpha_{k, n_{1}}\right]_{k \in \mathcal{K}, n_{1} \in \mathcal{N}_{1, k}}$

$$
\begin{array}{r}
\varphi_{k, n}(\mathbf{w}, \mathbf{v}, \boldsymbol{\alpha}) \triangleq \underbrace{\sum_{\bar{n} \in \mathcal{N}_{k} \backslash\{n\}}\left|\mathbf{h}_{k, k, n}^{H} \mathbf{w}_{k, \bar{n}}\right|^{2}+\left|\mathbf{h}_{k, k, n}^{H} \mathbf{v}_{k}\right|^{2}}_{\text {intracell interference }} \\
+\underbrace{\sum_{\bar{k} \in \mathcal{K} \backslash\{k\}} \sum_{\bar{n} \in \mathcal{N}_{\bar{k}}}\left|\mathbf{h}_{\bar{k}, k, n}^{H} \mathbf{w}_{\bar{k}, \bar{n}}\right|^{2}+\sum_{\bar{k} \in\{k\}}\left|\mathbf{h}_{\bar{k}, k, n}^{H} \mathbf{v}_{\bar{k}}\right|^{2}}_{\text {intercell interference }}
\end{array}
$$

$\sigma_{a}^{2}$ and $\sigma_{c}^{2}$ are respectively the antenna and circuit noise variances and

$$
\bar{\alpha}_{k, n}=\left\{\begin{array}{ll}
\alpha_{k, n}, & n \in \mathcal{N}_{1, k} \\
1, & n \notin \mathcal{N}_{1, k}
\end{array} .\right.
$$

The energy harvested at $\mathrm{UE}(k, n)$ is given by

$$
E_{k, n}\left(\mathbf{w}, \mathbf{v}, \boldsymbol{\alpha}_{k, n}\right) \triangleq \zeta_{k, n}\left(1-\alpha_{k, n}\right) p_{k, n}(\mathbf{w}, \mathbf{v}),
$$

where

$p_{k, n}(\mathbf{w}, \mathbf{v}) \triangleq \sum_{\bar{k} \in \mathcal{K}} \sum_{\bar{n} \in \mathcal{N}_{\bar{k}}}\left|\mathbf{h}_{\bar{k}, k, n}^{H} \mathbf{w}_{\bar{k}, \bar{n}}\right|^{2}+\sum_{\bar{k} \in \mathcal{K}}\left|\mathbf{h}_{\bar{k}, k, n}^{H} \mathbf{v}_{\bar{k}}\right|^{2}+\sigma_{a}^{2}$,

and the constant $\zeta_{k, n} \in(0,1)$ denotes the efficiency of energy conversion at the $\mathrm{EH}$ receiver.

In this paper, we consider the worst-case scenario, where for UE $(k, n)$, the zone-1 UEs $\left(k, \bar{n}_{1}\right) \forall \bar{n}_{1} \in \mathcal{N}_{1, k} \backslash n$ in cell $k$ cooperate to form a single eavesdropper $\left(k, n^{\prime}\right)$ with $N_{\mathrm{ev}}$ antennas, where $N_{\mathrm{ev}}=N_{1, k}-1$ or $N_{\mathrm{ev}}=N_{1, k}$, for the cases if $n \in \mathcal{N}_{1, k}$ or $n \notin \mathcal{N}_{1, k}$, respectively. In what follows, we use $\operatorname{EV}\left(k, n^{\prime}\right)$ to denote eavesdropper $\left(k, n^{\prime}\right), \forall k \in \mathcal{K}$ and $n^{\prime} \in \mathcal{N}_{k}$, which tries to eavesdrop on the received signal of UE $(k, n)$. Thus using the composite channel $\mathcal{H}_{\bar{k}, k, n^{\prime}} \triangleq$

$$
\begin{cases}{\left[\mathbf{h}_{\bar{k}, k, 1}, \ldots, \mathbf{h}_{\bar{k}, k, n-1}, \mathbf{h}_{\bar{k}, k, n+1}, \ldots, \mathbf{h}_{\bar{k}, k, N_{1, k}}\right],} & n \in \mathcal{N}_{1, k} \\ {\left[\mathbf{h}_{\bar{k}, k, 1}, \ldots, \mathbf{h}_{\bar{k}, k, N_{1, k}}\right],} & n \notin \mathcal{N}_{1, k},\end{cases}
$$

the received SINR corresponding to the signal intended for the $\mathrm{UE}(k, n)$ at the $\mathrm{EV}\left(k, n^{\prime}\right)$ is given by

$$
\mathrm{SINR}-\mathrm{EV}_{k, n^{\prime}} \triangleq \frac{\left\|\boldsymbol{\mathcal { H }}_{k, k, n^{\prime}}^{H} \mathbf{w}_{k, n}\right\|^{2}}{q_{k, n^{\prime}}(\mathbf{w}, \mathbf{v})}
$$

where $q_{k, n^{\prime}}(\mathbf{w}, \mathbf{v}) \triangleq$

$\sum_{\bar{k} \in \mathcal{K} \backslash\{k\}} \sum_{\bar{n} \in \mathcal{N}_{\bar{k}}}\left\|\mathcal{H}_{\bar{k}, k, n^{\prime}}^{H} \mathbf{w}_{\bar{k}, \bar{n}}\right\|^{2}+\sum_{\bar{k} \in \mathcal{K}}\left\|\mathcal{H}_{\bar{k}, k, n^{\prime}}^{H} \mathbf{v}_{\bar{k}}\right\|^{2}+N_{\mathrm{ev}} \sigma_{a}^{2}$

Our aim is to jointly optimize the transmit beamforming vectors $\mathbf{w}_{k, n}$, the artificial noise vectors $\mathbf{v}_{k}$, and the PS ratios $\alpha_{k, n_{1}}$ for all $k \in \mathcal{K}, n \in \mathcal{N}_{k}, n_{1} \in \mathcal{N}_{1, k}$. The max-min SINR problem is then formulated as

$$
\begin{aligned}
& \max _{\substack{\mathbf{w}_{k, n}, \mathbf{v}_{k} \in \mathbb{C}^{M \times 1} \\
\alpha_{k, n_{1}} \in(0,1)}} F(\mathbf{w}, \mathbf{v}, \boldsymbol{\alpha}) \triangleq \min _{k \in \mathcal{K}, n \in \mathcal{N}_{k}} f_{k, n}(\mathbf{w}, \mathbf{v}, \boldsymbol{\alpha}) \\
& \text { s.t. } \quad \sum_{n \in \mathcal{N}_{k}}\left\|\mathbf{w}_{k, n}\right\|^{2}+\left\|\mathbf{v}_{k}\right\|^{2} \leq P_{k}^{\max }, \forall k \in \mathcal{K} \\
& \quad \sum_{k \in \mathcal{K}} \sum_{n \in \mathcal{N}_{k}}\left\|\mathbf{w}_{k, n}\right\|^{2}+\sum_{k \in \mathcal{K}}\left\|\mathbf{v}_{k}\right\|^{2} \leq P^{\max } \\
& p_{k, n}(\mathbf{w}, \mathbf{v})-\frac{e_{k, n}^{\min }}{\zeta_{k, n}\left(1-\alpha_{k, n}\right)} \geq 0, \forall k \in \mathcal{K}, n \in \mathcal{N}_{1, k}, \\
& q_{k, n^{\prime}}(\mathbf{w}, \mathbf{v}) \geq\left\|\mathcal{H}_{k, k, n^{\prime}}^{H} \mathbf{w}_{k, n}\right\|^{2} / c_{k, n}^{\max }, \forall k \in \mathcal{K}, n \in \mathcal{N}_{k} .
\end{aligned}
$$

Constraint (5b) caps the total transmit power of each BS $k$ at a predefined value $P_{k}^{\max }$. Constraint $(5 \mathrm{c})$ ensures that the total transmit power of the network does not exceed the allowable budget $P^{\max }$. Constraint $(5 \mathrm{~d})$ requires that the minimum energy harvested by $\mathrm{UE}(k, n)$ is greater than some 
target threshold $e_{k, n}^{\min }$. Constraint (5e) requires the received SINR by EV $\left(k, n^{\prime}\right)$ be smaller than some upper limit SINR $c_{k, n}^{\max }$. While (5b) and (5c) are convex, the objective (5a) is not concave and the constraint (5d) is not convex due to the strong coupling between $\mathbf{w}_{k, n}, \mathbf{v}_{k}$, and $\alpha_{k, n_{1}}$ in both the SINR-UE and $\mathrm{EH}$ expressions [see (1) and (3)]. Moreover, constraint (5e) is nonconvex in its current form, whereas (5a) is also nonsmooth due to the minimization operator. In summary, (5) is a nonconvex nonsmooth function optimization subject to nonconvex constraints, for which even finding a feasible solution to (5b)-(5e) is not an easy task.

\section{ITERATIVE OPTIMIZATION BASED SOLUTION}

Upon defining $\mathbf{W}_{k, n} \triangleq \mathbf{w}_{k, n} \mathbf{w}_{k, n}^{H} \succcurlyeq \mathbf{0}, \mathbf{V}_{k} \triangleq \mathbf{v}_{k} \mathbf{v}_{k}^{H} \succcurlyeq \mathbf{0}$, and $\mathbf{H}_{k, k, n} \triangleq \mathbf{h}_{k, k, n} \mathbf{h}_{k, k, n}^{H}$ and introducing a new variable $\gamma$, problem (5) is recast as:

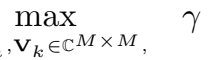

$$
\begin{aligned}
& \alpha_{k, n_{1}} \in(0,1), \gamma \\
& \text { s.t. } \frac{1}{\gamma} \operatorname{Tr}\left\{\mathbf{H}_{k, k, n} \mathbf{W}_{k, n}\right\}-\sum_{\bar{n} \in \mathcal{N}_{k} \backslash\{n\}} \operatorname{Tr}\left\{\mathbf{H}_{k, k, n} \mathbf{W}_{k, \bar{n}}\right\} \\
& -\sum_{\bar{k} \in \mathcal{K} \backslash\{k\}} \sum_{\bar{n} \in \mathcal{N}_{\bar{k}}} \operatorname{Tr}\left\{\mathbf{H}_{\bar{k}, k, n} \mathbf{W}_{\bar{k}, \bar{n}}\right\}-\sum_{\bar{k} \in \mathcal{K}} \operatorname{Tr}\left\{\mathbf{H}_{\bar{k}, k, n} \mathbf{V}_{\bar{k}}\right\} \\
& \geq \sigma_{a}^{2}+\frac{\sigma_{c}^{2}}{\bar{\alpha}_{k, n}}, \forall k \in \mathcal{K}, n \in \mathcal{N}_{k} \\
& \sum_{n \in \mathcal{N}_{k}} \operatorname{Tr}\left\{\mathbf{W}_{k, n}\right\}+\operatorname{Tr}\left\{\mathbf{V}_{k}\right\} \leq P_{k}^{\max }, \forall k \in \mathcal{K} \\
& \sum_{k \in \mathcal{K}} \sum_{n \in \mathcal{N}_{k}} \operatorname{Tr}\left\{\mathbf{W}_{k, n}\right\}+\sum_{k \in \mathcal{K}} \operatorname{Tr}\left\{\mathbf{V}_{k}\right\} \leq P^{\max } \\
& \sum_{\bar{k} \in \mathcal{K}} \sum_{\bar{n} \in \mathcal{N}_{\bar{k}}} \operatorname{Tr}\left\{\mathbf{H}_{\bar{k}, k, n} \mathbf{W}_{\bar{k}, \bar{n}}\right\}+\sum_{\bar{k} \in \mathcal{K}} \operatorname{Tr}\left\{\mathbf{H}_{\bar{k}, k, n} \mathbf{V}_{\bar{k}}\right\} \\
& \geq \frac{e_{k, n}^{\min }}{\zeta_{k, n}\left(1-\alpha_{k, n}\right)}-\sigma_{a}^{2}, \forall k \in \mathcal{K}, n \in \mathcal{N}_{1, k} \\
& \operatorname{Tr}\left\{\mathcal{H}_{k, k, n^{\prime}} \mathcal{H}_{k, k, n^{\prime}}^{H} \mathbf{W}_{k, n}\right\} \\
& -c_{k, n}^{\max } \sum_{\bar{k} \in \mathcal{K} \backslash\{k\}} \sum_{\bar{n} \in \mathcal{N}_{\bar{k}}} \operatorname{Tr}\left\{\mathcal{H}_{\bar{k}, k, n^{\prime}} \boldsymbol{\mathcal { H }}_{\bar{k}, k, n^{\prime}}^{H} \mathbf{W}_{\bar{k}, \bar{n}}\right\} \\
& -c_{k, n}^{\max } \sum_{\bar{k} \in \mathcal{K} \backslash\{k\}} \operatorname{Tr}\left\{\mathcal{H}_{\bar{k}, k, n^{\prime}} \mathcal{H}_{\bar{k}, k, n^{\prime}}^{H} \mathbf{V}_{\bar{k}}\right\} \\
& \leq c_{k, n}^{\max }(N-1) \sigma_{a}^{2}, \forall k \in \mathcal{K}, n \in \mathcal{N}_{k} \\
& \mathbf{W}_{k, n} \succcurlyeq \mathbf{0}, \forall k \in \mathcal{K}, n \in \mathcal{N}_{k} \\
& \operatorname{rank}\left(\mathbf{W}_{k, n}\right)=1, \operatorname{rank}\left(\mathbf{V}_{k}\right)=1, \forall k \in \mathcal{K}, n \in \mathcal{N}_{k} .
\end{aligned}
$$

Let us also denote $\mathbf{W} \triangleq\left[\mathbf{W}_{k, n}\right]_{k \in \mathcal{K}, n \in \mathcal{N}_{k}}$ and $\mathbf{V} \triangleq\left[\mathbf{V}_{k}\right]_{k \in \mathcal{K}}$. Since $\frac{1}{1-\alpha_{k, n_{1}}}$ is convex on $\alpha_{k, n_{1}} \in(0,1)$, constraints (6c)$(6 \mathrm{~g})$ are convex in $(\mathbf{W}, \mathbf{V}, \boldsymbol{\alpha}, \gamma)$. Now, in order to solve (6), we have to deal with the nonconvex constraints (6b) and (6h). For a fixed value of $\gamma,(6 \mathrm{~b})$ is convex in $\left(\mathbf{W}, \mathbf{V}, \alpha_{k, n_{1}}\right)$ because $\frac{1}{\alpha_{k, n_{1}}}$ is convex on $\alpha_{k, n_{1}} \in(0,1)$. By fixing $\gamma$ and ignoring the difficult rank-one constraint $(6 \mathrm{~h}),(6)$ becomes a feasibility (convex) semidefinite relaxation (SDR) (6b)-(6g) which can be efficiently solved. Note that the optimal value of $\gamma$ can be found via a bisection search because (6b) is the only constraint that involves $\gamma$ and it is monotonic in $\gamma$. The optimization process is repeated until $(\mathbf{W}, \mathbf{V}, \boldsymbol{\alpha}, \gamma)$ converges to $\left(\mathbf{W}^{\star}, \mathbf{V}^{\star}, \boldsymbol{\alpha}^{\star}, \gamma^{\star}\right), \forall k \in \mathcal{K}, n \in \mathcal{N}_{k}, n_{1} \in \mathcal{N}_{1, k}$, in which case $(6 \mathrm{a})-(6 \mathrm{~g})$ is solved.

If $\operatorname{rank}\left(\mathbf{W}_{k, n}^{\star}\right)=1, \forall k \in \mathcal{K}, n \in \mathcal{N}_{k}$ and $\operatorname{rank}\left(\mathbf{V}_{k}^{\star}\right)=$ $1, \forall k \in \mathcal{K}$, the rank-one constraint (6h) is automatically satisfied. Thus, the optimal beamforming vectors $\mathbf{w}_{k, n}^{\star}$ and $\mathbf{v}_{k}^{\star}$ of (5) can then be recovered from the eigenvalue decomposition (EVD) of $\mathbf{W}_{k, n}^{\star}$ and $\mathbf{V}_{k}^{\star}$, respectively, and its optimal PS ratio is simply $\alpha_{k, n_{1}}^{\star}$. However, the problem arises when $\operatorname{rank}\left(\mathbf{W}_{k, n}^{\star}\right)>1$ or $\operatorname{rank}\left(\mathbf{V}_{k}^{\star}\right)>1$ for some UE $(k, n)$. Unfortunately, we find it to be true quite often and thus, $\gamma^{\star}$ serves only as an upper bound to the optimum of (6) and hence of (5). Our simulation results in Sec. IV confirms that the solution of (6a)-(6g) in a three-cell network has $\operatorname{rank}\left(\mathbf{W}_{k, n}^{\star}\right)>1$ or $\operatorname{rank}\left(\mathbf{V}_{k}^{\star}\right)>1$ for some $(k, n)$ in about $88.5 \%$ of the time. This clearly shows that solving the feasibility SDR (6a)$(6 \mathrm{~g})$ alone is not acceptable to recover the optimal solution $\left(\mathbf{w}_{k, n}^{\star}, \alpha_{k, n}^{\star}\right)$ of (5). The current approach in the literature is to use randomization to extract the beamforming vectors $\left\{\mathbf{w}_{k, n}, \mathbf{v}_{k}\right\}$ from $\left\{\mathbf{W}_{k, n}^{\star}, \mathbf{V}_{k}^{\star}\right\}$ [8]. However, as shown in this paper, the performance of randomization is worse than the upper bound SDR. In the following, we propose two methods to solve this problem.

\section{A. SDP-based Iterative Spectral Optimization}

For any matrix $\mathbf{X} \succcurlyeq \mathbf{0}$, it is true that $\operatorname{Tr}\{\mathbf{X}\}-\lambda_{\max }\{\mathbf{X}\} \geq$ 0 , where $\lambda_{\max }\{\cdot\}$ stands for the maximum eigenvalue of a matrix. Thus, rank-one constraints (6h) can be expressed as $\operatorname{Tr}\{\mathbf{X}\}-\lambda_{\max }\{\mathbf{X}\}=0 \forall \mathbf{X} \in\left\{\mathbf{W}_{k, n}, \mathbf{V}_{k}\right\}$. For a given $\gamma$, following the approach of [9] we reformulate problem (6) as the following program:

$$
\begin{aligned}
& \min _{\substack{\mathbf{W}_{k, n} \in \mathbb{C}^{M \times M} \\
\alpha_{k, n_{1}} \in(0,1)}} \tilde{F}(\mathbf{W}, \mathbf{V}) \triangleq \sum_{k \in \mathcal{K}} \sum_{n \in \mathcal{N}_{k}} \operatorname{Tr}\left\{\mathbf{W}_{k, n}\right\}-\lambda_{\max }\left\{\mathbf{W}_{k, n}\right\} \\
& \quad \text { s.t. } \quad(6 \mathrm{~b})-(6 \mathrm{~g}),
\end{aligned}
$$

which is the exact penalization of (6). From [13], we have that

$$
\begin{aligned}
& \lambda_{\max }\left\{\mathbf{X}_{k, n}\right\} \geq \lambda_{\max }\left\{\mathbf{W}_{k, n}\right\} \\
& \quad+\left(\mathbf{w}_{k, n}^{\max }\right)^{H}\left(\mathbf{X}_{k, n}-\mathbf{W}_{k, n}\right) \mathbf{w}_{k, n}^{\max }, \forall k \in \mathcal{K}, n \in \mathcal{N}_{k}
\end{aligned}
$$

for any $\mathbf{X}_{k, n} \succcurlyeq \mathbf{0}$ and $\mathbf{Y}_{k} \succcurlyeq \mathbf{0}$. As such, given some feasible $\mathbf{W}_{k, n}^{(\kappa)}$ and $\mathbf{V}_{k}^{(\kappa)}$ of (7) at iteration $\kappa$ with the corresponding maximum eigenvalue $\lambda_{\max }\left\{\mathbf{w}_{k, n}^{\max ,(\kappa)}\right\}$ and $\lambda_{\max }\left\{\mathbf{v}_{k}^{\max ,(\kappa)}\right\}$, respectively, and unit-norm eigenvector $\mathbf{w}_{k, n}^{\max ,(\kappa)}$ and $\mathbf{v}_{k}^{\max ,(\kappa)}$, respectively, it follows that

$$
\begin{aligned}
\tilde{F}^{(\kappa)}(\mathbf{W}, \mathbf{V}) \triangleq \sum_{k \in \mathcal{K}} \sum_{n \in \mathcal{N}_{k}} \operatorname{Tr}\left\{\mathbf{W}_{k, n}\right\}+\sum_{k \in \mathcal{K}} \operatorname{Tr}\left\{\mathbf{V}_{k}\right\} \\
-\lambda_{\max }\left\{\mathbf{W}_{k, n}^{(\kappa)}\right\}-\left(\mathbf{w}_{k, n}^{\max ,(\kappa)}\right)^{H}\left(\mathbf{W}_{k, n}-\mathbf{W}_{k, n}^{(\kappa)}\right) \mathbf{w}_{k, n}^{\max ,(\kappa)} \\
-\lambda_{\max }\left\{\mathbf{V}_{k}^{(\kappa)}\right\}-\left(\mathbf{v}_{k}^{\max ,(\kappa)}\right)^{H}\left(\mathbf{V}_{k}-\mathbf{V}_{k}^{(\kappa)}\right) \mathbf{v}_{k, n}^{\max ,(\kappa)} \\
\geq F(\mathbf{W}, \mathbf{V}), \forall \mathbf{W}, \mathbf{V}
\end{aligned}
$$

Thus, the following SDP

$$
\min _{\substack{\mathbf{w}_{k, n}, \mathbf{v}_{k} \in \mathbb{C}^{M} \times M \\ \alpha_{k, n_{1}} \in(0,1)}} \tilde{F}^{(\kappa)}(\mathbf{W}, \mathbf{V}) \quad \text { s.t. } \quad(6 \mathrm{~b})-(6 \mathrm{~g}) .
$$




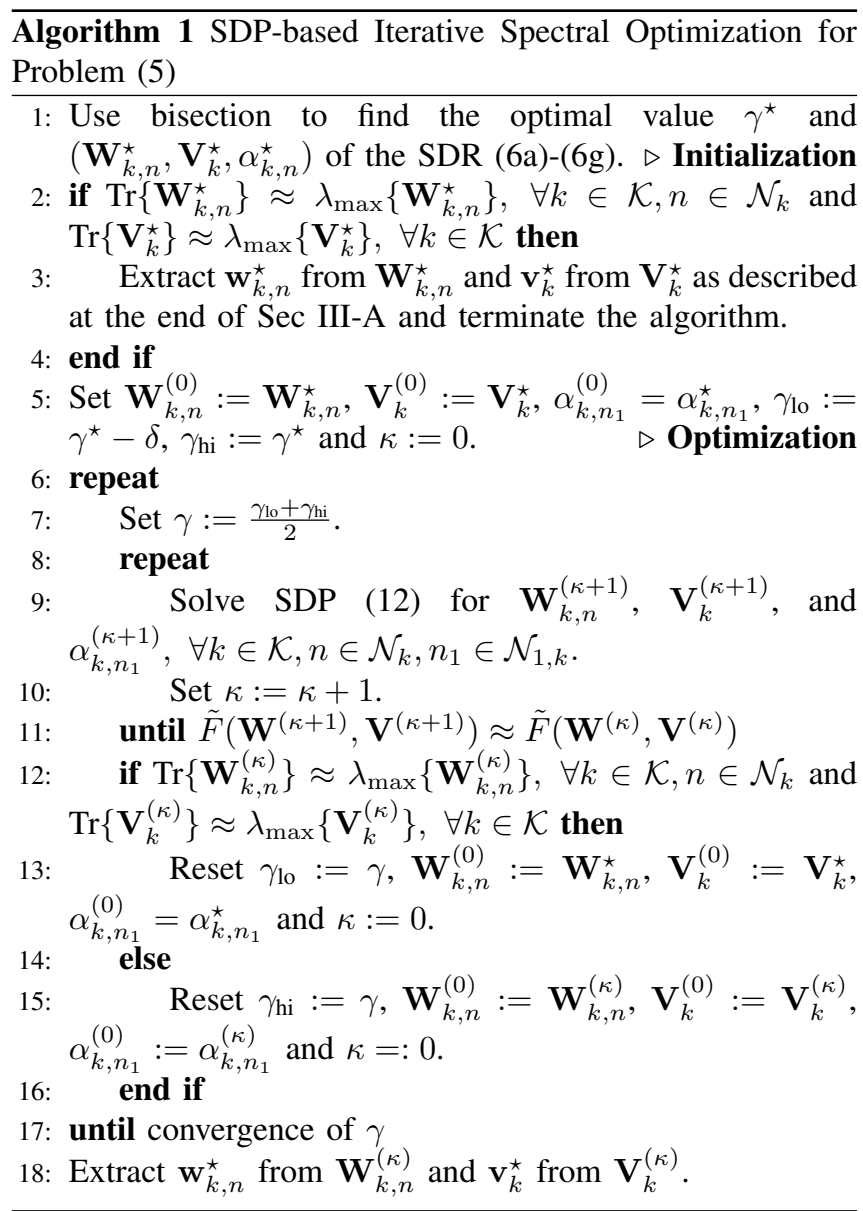

is a convex majorant minimization of the nonconvex program (7). Note that the optimal solution $\left\{\mathbf{W}^{(\kappa+1)}, \mathbf{V}^{(\kappa+1)}, \boldsymbol{\alpha}^{(\kappa+1)}\right\}$ of (10) is a better solution to (7) than $\left\{\mathbf{W}^{(\kappa)}, \mathbf{V}^{(\kappa)}, \boldsymbol{\alpha}^{(\kappa)}\right\}$ as

$$
\begin{aligned}
\tilde{F}\left(\mathbf{W}^{(\kappa+1)}, \mathbf{V}^{(\kappa+1)}\right) \leq \tilde{F}^{(\kappa)}\left(\mathbf{W}^{(\kappa+1)}, \mathbf{V}^{(\kappa+1)}\right) \\
<\tilde{F}^{(\kappa)}\left(\mathbf{W}^{(\kappa)}, \mathbf{V}^{(\kappa)}\right)=\tilde{F}\left(\mathbf{W}^{(\kappa)}, \mathbf{V}^{(\kappa)}\right)
\end{aligned}
$$

as far as $\left(\mathbf{W}_{k, n}^{(\kappa+1)}, \mathbf{V}_{k}^{(\kappa+1)}, \alpha_{k, n_{1}}^{(\kappa+1)}\right) \neq\left(\mathbf{W}_{k, n}^{(\kappa)}, \mathbf{V}_{k}^{(\kappa)}, \alpha_{k, n_{1}}^{(\kappa)}\right)$ for some $(k, n)$. Program (10) can be further simplified to:

$$
\begin{aligned}
\min _{\substack{\mathbf{w}_{k, n} \in \mathbb{C}^{M \times M} \\
\alpha_{k, n} \in(0,1)}} \sum_{k \in \mathcal{K}} \sum_{n \in \mathcal{N}_{k}} \operatorname{Tr}\left\{\mathbf{W}_{k, n}\right\}-\left(\mathbf{w}_{k, n}^{\max ,(\kappa)}\right)^{H} \mathbf{W}_{k, n} \mathbf{w}_{k, n}^{\max ,(\kappa)} \\
\quad+\sum_{k \in \mathcal{K}} \operatorname{Tr}\left\{\mathbf{V}_{k}\right\}-\left(\mathbf{v}_{k}^{\max ,(\kappa)}\right)^{H} \mathbf{V}_{k} \mathbf{v}_{k}^{\max ,(\kappa)} \\
\text { s.t. (6b) }-(6 \mathrm{~g}) .
\end{aligned}
$$

With (12), we propose to employ a bisection search in an outer loop to obtain the optimal value of $\gamma$.

In Algorithm 1, we propose an SDP-based iterative algorithm to solve the problem (5). We choose the initial solution $\left(\mathbf{W}_{k, n}^{(0)}, \mathbf{V}_{k}^{(0)}, \alpha_{k, n_{1}}^{(0)}\right)$ as the optimal solution $\left(\mathbf{W}_{k, n}^{\star}, \mathbf{V}_{k}^{\star}, \alpha_{k, n_{1}}^{\star}\right)$ of (6a)-(6g). Hence, $\mathbf{W}_{k, n}^{(0)}$ or $\mathbf{V}_{k}^{(0)}$ may not be of rank one, however, Optimization stage will ensure a rank-one solution. In the Optimization stage, the inner loop optimizes $\mathbf{W}_{k, n}, \mathbf{V}_{k}, \alpha_{k, n_{1}}, \forall k \in \mathcal{K}, n \in \mathcal{N}_{k}, n_{1} \in \mathcal{N}_{1, k}$ for a given value of $\gamma$ by solving exactly one convex SDP
(12) in each iteration. Once $\tilde{F}(\mathbf{W}, \mathbf{V})$ converges, the inner loop terminates and we determine the rank of the optimized beamforming matrices $\mathbf{W}_{k, n}^{(\kappa)}$ and $\mathbf{V}_{k}^{(\kappa)}$. If $\operatorname{Tr}\left\{\mathbf{W}_{k, n}^{(\kappa)}\right\} \approx$ $\lambda_{\max }\left\{\mathbf{W}_{k, n}^{(\kappa)}\right\}$ and $\operatorname{Tr}\left\{\mathbf{V}_{k}^{(\kappa)}\right\} \approx \lambda_{\max }\left\{\mathbf{V}_{k}^{(\kappa)}\right\}$, we update $\gamma_{\mathrm{lo}}:=\gamma$, and otherwise we set $\gamma_{\mathrm{hi}}:=\gamma$. The outer loop optimizes $\gamma$ via a simple bisection search. The upper and lower limits for the bisection search are set as $\gamma_{\mathrm{hi}}=\gamma^{\star}$ and $\gamma_{\mathrm{lo}}=\gamma^{\star}-\delta$, where $\delta>0$ and $\gamma^{\star}$ is the optimal upper bound obtained by solving (6a)-(6g) during the Initialization stage. After the convergence of $\gamma$, the optimal beamforming vectors $\mathbf{w}_{k, n}^{\star}$ and $\mathbf{v}_{k}^{\star}$ of problem (5) are recovered from the optimal matrices $\mathbf{W}_{k, n}^{\mathrm{opt}} \triangleq \mathbf{W}_{k, n}^{(\kappa)}$ and $\mathbf{V}_{k}^{\mathrm{opt}} \triangleq \mathbf{V}_{k}^{(\kappa)}$, respectively, according to $\mathbf{w}_{k, n}^{\star}=\left(\lambda_{\max }\left\{\mathbf{W}_{k, n}\right\}\right)^{1 / 2} \mathbf{w}_{k, n}^{\max }$ and $\mathbf{v}_{k}^{\star}=\left(\lambda_{\max }\left\{\mathbf{V}_{k}\right\}\right)^{1 / 2} \mathbf{v}_{k}^{\max }$.

\section{B. QP-based Iterative Difference-of-Convex-Functions (DC) Optimization}

In what follows, we will show that it is possible to solve the original problem (5) directly in the vector variables $\mathbf{w}_{k, n} \in$ $\mathbb{C}^{M \times 1}$ and $\mathbf{v}_{k} \in \mathbb{C}^{M \times 1}$ via quadratic programming (QP), also avoiding the bisection search.

Proposition 1: For $\pi(\mathbf{x})=p(\mathbf{x}) / q(\mathbf{x})$ with positive convex quadratic function $p$ and strictly positive and convex function $q(\mathbf{x})$, it is true that [14]

$$
\begin{aligned}
\pi(\mathbf{x}) \geq \tilde{\pi}(\mathbf{x}): & =(p(\bar{x})+\langle\nabla p(\bar{x}), \mathbf{x}-\bar{x}\rangle) / q(\bar{x}) \\
& -p(\bar{x})(q(\mathbf{x})-q(\bar{x})) / q^{2}(\bar{x}) \quad \forall(\mathbf{x}, \bar{x}),
\end{aligned}
$$

and $\pi(\bar{x})=\tilde{\pi}(\bar{x})$, where $\nabla$ defines the first order differential operator, and $\langle\mathbf{x}, \mathbf{y}\rangle \triangleq \mathbf{x}^{H} \mathbf{y}$.

Applying Proposition 1 at $\left\{\mathbf{w}_{k, n}^{(\kappa)}, \mathbf{v}_{k}^{(\kappa)}\right\}$ given from $\kappa$-th iteration, the following program provides a minorant maximization for the nonconvex program (5):

$$
\begin{aligned}
& \max _{\substack{\mathbf{v}_{k}, \mathbf{w}_{k, n} \in \mathbb{C}^{M \times 1} \\
\alpha_{k, n_{1}} \in(0,1)}} \min _{\substack{\mathcal{K}_{n} \in \mathcal{N}_{k} \\
\text { s.t. }}} f_{k, n}^{(\kappa)}(\mathbf{w}, \mathbf{v}, \boldsymbol{\alpha}) \\
& \text { (k), }(\mathbf{w}, \mathbf{v})-\frac{e_{k, n}^{\min }}{\zeta_{k, n}\left(1-\alpha_{k, n}\right)} \geq 0, \forall k \in \mathcal{K}, n \in \mathcal{N}_{1, k},
\end{aligned}
$$

$$
\left\|\mathcal{H}_{k, k, n^{\prime}}^{H} \mathbf{w}_{k, n}\right\|^{2} / c_{k, n}^{\max }-q_{k, n^{\prime}}^{(\kappa)}(\mathbf{w}, \mathbf{v}) \leq 0 \forall k \in \mathcal{K}, n \in \mathcal{N}_{k}
$$

$(5 b),(5 c)$,

where

$$
\begin{aligned}
& p_{k, n}^{(\kappa)}(\mathbf{w}, \mathbf{v})=p_{k, n}\left(\mathbf{w}^{(\kappa)}, \mathbf{v}^{(\kappa)}\right) \\
& \quad+2 \sum_{\bar{k} \in \mathcal{K}} \sum_{\bar{n} \in \mathcal{N}_{\bar{k}}} \Re\left\{\left(\mathbf{w}_{\bar{k}, \bar{n}}^{(\kappa)}\right)^{H} \mathbf{h}_{\bar{k}, k, n} \mathbf{h}_{\bar{k}, k, n}^{H}\left(\mathbf{w}_{\bar{k}, \bar{n}}-\mathbf{w}_{\bar{k}, \bar{n}}^{(\kappa)}\right)\right\} \\
& \quad+2 \sum_{\bar{k} \in \mathcal{K}} \Re\left\{\left(\mathbf{v}_{\bar{k}}^{(\kappa)}\right)^{H} \mathbf{h}_{\bar{k}, k, n} \mathbf{h}_{\bar{k}, k, n}^{H}\left(\mathbf{v}_{\bar{k}}-\mathbf{v}_{\bar{k}}^{(\kappa)}\right)\right\}, \quad(16) \\
& q_{k, n^{\prime}}^{(\kappa)}(\mathbf{w}, \mathbf{v})=q_{k, n^{\prime}}\left(\mathbf{w}^{(\kappa)}, \mathbf{v}^{(\kappa)}\right) \\
& +2 \sum_{\bar{k} \in \mathcal{K} \backslash\{k\}} \sum_{\bar{n} \in \mathcal{N}_{\bar{k}}} \Re\left\{\left(\mathbf{w}_{\bar{k}, \bar{n}}^{(\kappa)}\right)^{H} \mathcal{H}_{\bar{k}, k, n^{\prime}} \mathcal{H}_{\bar{k}, k, n^{\prime}}^{H}\left(\mathbf{w}_{\bar{k}, \bar{n}}-\mathbf{w}_{\bar{k}, \bar{n}}^{(\kappa)}\right)\right\} \\
& +2 \sum_{\bar{k} \in \mathcal{K}} \Re\left\{\left(\mathbf{v}_{\bar{k}}^{(\kappa)}\right)^{H} \mathcal{H}_{\bar{k}, k, n^{\prime}} \mathcal{H}_{\bar{k}, k, n^{\prime}}^{H}\left(\mathbf{v}_{\bar{k}}-\mathbf{v}_{\bar{k}}^{(\kappa)}\right)\right\},
\end{aligned}
$$




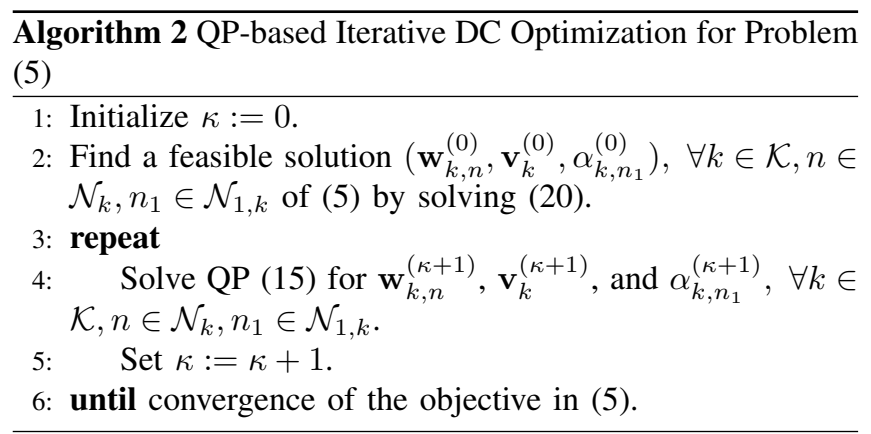

and

$$
\begin{aligned}
& f_{k, n}^{(\kappa)}(\mathbf{w}, \mathbf{v}, \boldsymbol{\alpha})=f_{k, n}\left(\mathbf{w}^{(\kappa)}, \mathbf{v}^{(\kappa)}, \boldsymbol{\alpha}^{(\kappa)}\right) \\
& +2 \Re\left\{\frac{\left(\mathbf{w}_{k, n}^{(\kappa)}\right)^{H} \mathbf{h}_{k, k, n} \mathbf{h}_{k, k, n}^{H}\left(\mathbf{w}_{k, n}-\mathbf{w}_{k, n}^{(\kappa)}\right)}{\varphi_{k, n}\left(\mathbf{w}^{(\kappa)}, \mathbf{v}^{(\kappa)}, \boldsymbol{\alpha}^{(\kappa)}\right)}\right\} \\
& -\frac{\left|\mathbf{h}_{k, k, n}^{H} \mathbf{w}_{k, n}^{(\kappa)}\right|^{2}\left(\varphi_{k, n}(\mathbf{w}, \mathbf{v}, \boldsymbol{\alpha})-\varphi_{k, n}\left(\mathbf{w}^{(\kappa)}, \mathbf{v}^{(\kappa)}, \boldsymbol{\alpha}^{(\kappa)}\right)\right)}{\left(\varphi_{k, n}\left(\mathbf{w}^{(\kappa)}, \mathbf{v}^{(\kappa)}, \boldsymbol{\alpha}^{(\kappa)}\right)\right)^{2}}
\end{aligned}
$$

For initialization, one can find a feasible solution of (5) by solving

$$
\begin{array}{r}
\max _{\mathbf{w}, \mathbf{v}, \boldsymbol{\alpha}} \min _{k, n}\left\{p_{k, n_{1}}(\mathbf{w}, \mathbf{v})-e_{k, n_{1}}^{\min } / \zeta_{k, n_{1}}\left(1-\alpha_{k, n_{1}}\right),\right. \\
\left.q_{k, n^{\prime}}(\mathbf{w}, \mathbf{v})-\left\|\boldsymbol{H}_{k, k, n^{\prime}}^{H} \mathbf{w}_{k, n}\right\|^{2} / c_{k, n}^{\max }\right\}:(5 \mathbf{b}),
\end{array}
$$

for which there is no issue in finding a feasible solution since constraints (5b) and (5c) are convex. For this, at each iteration, one can use Proposition 1 for lower bounding each function in (19) as follows. Initialized from $\left(\mathbf{w}^{(0)}, \mathbf{v}^{(0)}, \boldsymbol{\alpha}^{(0)}\right)$, one solves

$$
\begin{array}{r}
\max _{\mathbf{w}, \mathbf{v}, \boldsymbol{\alpha}} \min _{k, n}\left\{p_{k, n_{1}}^{(\kappa)}(\mathbf{w}, \mathbf{v})-e_{k, n_{1}}^{\min } / \zeta_{k, n_{1}}\left(1-\alpha_{k, n_{1}}\right),\right. \\
\left.q_{k, n^{\prime}}^{(\kappa)}(\mathbf{w}, \mathbf{v})-\left\|\boldsymbol{H}_{k, k, n^{\prime}}^{H} \mathbf{w}_{k, n}\right\|^{2} / c_{k, n}^{\max }\right\}:(5 \mathbf{b}),
\end{array}
$$

The iterative process stops whenever the value of (19) is more than or equal 0. In Algorithm 2, we propose a QP-based iterative algorithm to solve the max-min SINR problem (5). The initial solution $\mathbf{w}^{(0)} \triangleq\left[\mathbf{w}_{k, n}^{(0)}\right]_{k \in \mathcal{K}, n \in \mathcal{N}_{k}}, \mathbf{v}^{(0)} \triangleq\left[\mathbf{v}_{k}^{(0)}\right]_{k \in \mathcal{K}}$, and $\boldsymbol{\alpha}^{(0)} \triangleq\left[\alpha_{k, n_{1}}^{(0)}\right]_{k \in \mathcal{K}, n_{1} \in \mathcal{N}_{1, k}}$ is obtained by iteratively solving (20). We proceed to solving one QP (15) in each iteration, the solution of which is used to improve the objective value in the next iteration.

\section{NUMERICAL EXAMPLES}

Fig. 1 shows the network topology with $K=3$ cells and $N=N_{k}=4, \forall k \in \mathcal{K}$ UEs per cell that we use in our numerical examples. Out of the 4 users in each cell, two are placed inside the inner-circle and two inside the outer-zone, i.e., $N_{1, k}=N_{2, k}=2, \forall k$. We set the cell radius as $40 \mathrm{~m}$ and the BS-to-UE distance is $7 \mathrm{~m}$ and $20 \mathrm{~m}$ in the inner and outer zone of each cell, respectively. The minimum energy harvesting requirement for UEs inside inner circle is $e_{k, n_{1}}^{\min }=$ $20 \mathrm{dBm} \forall, k, n_{1}$. We set the path loss exponent as $\beta=3$. For small-scale fading, we generate the Rician fading channel

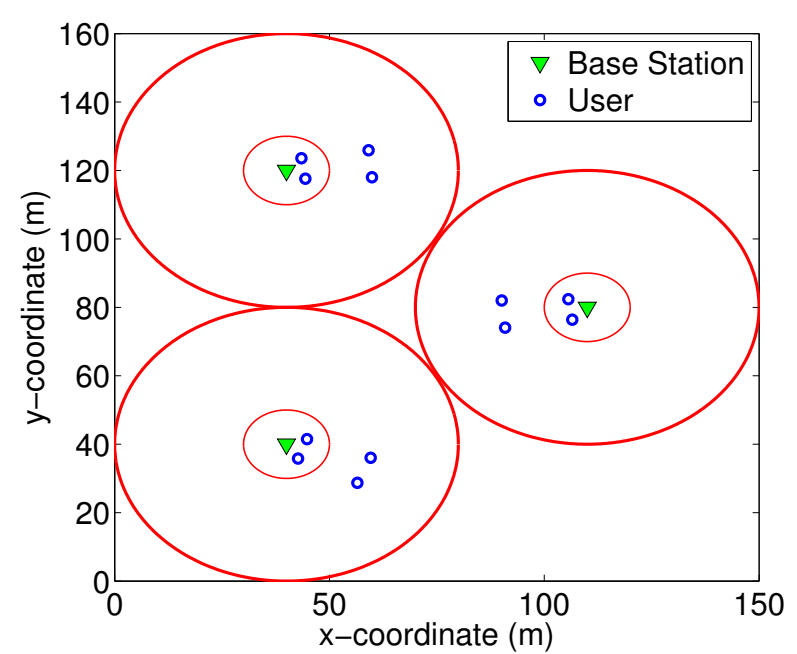

Fig. 1. Topology of the multicell network used in numerical examples

according to Rician factor, $K_{R}=10 \mathrm{~dB}$ [15]. For simplicity and without loss of generality, we assume that $\zeta_{k, n}=\zeta, \forall k, n$. In all simulations, we set $\zeta=0.5, \sigma_{a}^{2}=-90 \mathrm{dBm}, \sigma_{c}^{2}=-90$ $\mathrm{dBm}$, and $P^{\max }=34 \mathrm{dBm}$. For the SDR method, we set the upper and lower limits for the bisection search as $\gamma_{\mathrm{hi}}=1,000$ (i.e., $30 \mathrm{~dB}$ ) and $\gamma_{\mathrm{lo}}=0$. For Algorithm 1, we choose $\delta=1$ after fine tuning.

Fig. 2 illustrates the convergence of Algorithms 1 and 2 versus the SDR approach. In this figure, each iteration corresponds to solving one SDP (6a) $-(6 \mathrm{~g})$ or one SDP (12) in Algorithm 1, one QP (15) in Algorithm 2, and one SDP (6a)$(6 \mathrm{~g})$ in the SDR approach. Here, the initial SNR values for both Algorithm 1 and the SDR approach are similar because Algorithm 1 also solves the SDR in order to generate a feasible initial solution. After 15 iterations, the SDR solution converges and Algorithm 1 takes that converged solution to execute its Steps 6-17. The remaining iterations in the curve for Algorithm 1 illustrate the number of SDP (12) solved in Steps 6-17 of that algorithm. Algorithm 2 takes a single iteration during the initialization stage and then converges quickly after 10 iterations to the optimal SINR.

We observe that solving an SDR fails to deliver a rank-one solution in $88.5 \%$ of the time on average. In our simulations, we establish that a matrix is only of rank one if the magnitude of its second largest eigenvalue is less than $\rho=1 / 200$ of that of its largest eigenvalue. Since this criterion is much more relaxed than conventionally where $\rho$ is much smaller, it ensures that a rank-one matrix is not mistaken. Fig. 3 plots the maximized minimum UE SINR for different values of $P_{k}^{\max }=\{24, \ldots, 30\} \mathrm{dBm}$, while using $M=4$ and $M=5$ antennas. It is clear that the performance of the SDRrandomization method is away from the optimal upper bound given by SDR. Increasing the number of antennas from $M=4$ to $M=5$ further increases the performance gap between upper bound and SDR-randomization method. In contrast, the performance of our proposed Algorithms 1 and 2 is quite close to this bound, under different network setups. As shown in Table I, we observe that for $M=5$ antennas setup, SDR 


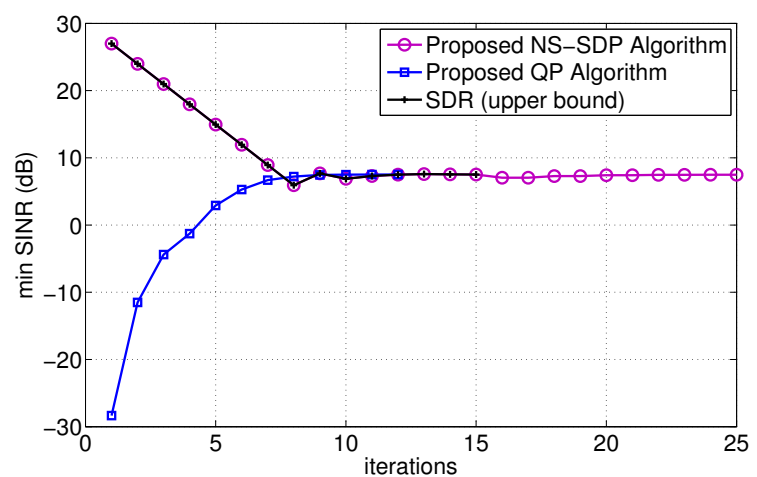

Fig. 2. Convergence of proposed Algorithms for $M=4$ and $P_{k}^{\max }=$ $24 \mathrm{dBm})$

approach always fails to guarantee a rank-one $\mathbf{W}_{k, n}^{\star}$ solution. In comparison, Algorithm 1 guarantees to always return rankone matrix solutions. Algorithm 2 of course directly gives vector $\mathbf{w}_{k, n}^{\star}$ because no matrix optimization is involved.

TABLE I

Percentage of time When RANK $\left\{\mathbf{W}_{k, n}^{\star}\right\}>1$ In Problem (5)

\begin{tabular}{|c||c|c|c|c|}
\hline Algorithms / $P_{k}^{\max }$ & $=24 \mathrm{dBm}$ & $26 \mathrm{dBm}$ & $28 \mathrm{dBm}$ & $30 \mathrm{dBm}$ \\
\hline \hline SDR (M=4) & $76 \%$ & $77 \%$ & $82 \%$ & $74 \%$ \\
\hline SDR (M=5) & $100 \%$ & $100 \%$ & $100 \%$ & $100 \%$ \\
\hline Alg. 1 $(\mathrm{M}=4,5)$ & $0 \%$ & $0 \%$ & $0 \%$ & $0 \%$ \\
\hline Alg. 2 $(\mathrm{M}=4,5)$ & $0 \%$ & $0 \%$ & $0 \%$ & $0 \%$ \\
\hline
\end{tabular}

1) Complexity Analysis: On average, in order to acquire the optimal solution, the proposed Algorithm 1 solves 15 and 9.1 semi-definite programs (SDPs) during initialization and optimization stage (including inner and outer optimization loops for optimization stage), respectively, the proposed Algorithm 2 solves 1 and 12.2 quadratic programs (QPs) during initialization and optimization stages, respectively, and the upper bound SDR approach solves for 15 SDPs. Our simulation analysis shows that the average execution time per iteration is approximately the same for SDP and QP, yielding almost similar computational complexity for Algorithm 2 and SDR approach and almost double computational for Algorithm 1. However, it is worth mentioning unlike the SDR-based solution, the optimal beamforming matrices of the proposed Algorithm 1 are always of rank one. Furthermore, the optimal SINR of the proposed Algorithms 1 and 2 approximately achieve the upper bound provided by SDR.

\section{Conclusion}

In this paper, we have addressed the joint design of secure beamforming and receive power splitting in a dense multi-cell network. Due to the unavoidable rank-one matrix constraints, the conventional approach is not applicable in this case. We have therefore proposed two new iterative optimization approaches that offer maximized minimum SINR among all UEs. The optimal solutions provided by our SDP-based spectral optimization and QP-based DCI algorithms approach the theoretical bound. Significantly, they do so with a complexity

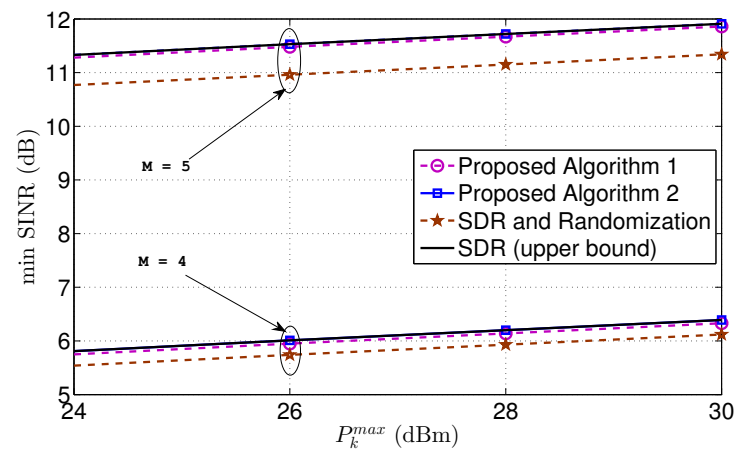

Fig. 3. Maximized minimum UE SINR for $M=4$ and $P^{\max }=34 \mathrm{dBm}$ )

that is comparable to that by existing methods. The merits of our proposed algorithms have been confirmed through numerical examples with realistic parameters.

\section{REFERENCES}

[1] H. Zhang, C. Li, Y. Huang, and L. Yang, "Secure beamforming for SWIPT in multiuser MISO broadcast channels with confidential messages," IEEE Commun. Lett., vol. PP, no. 99, pp. 1-1, 2015.

[2] W. Wu and B. Wang, "Robust downlink beamforming design for multiuser MISO communication system with SWIPT," in Proc. IEEE ICC, June 2015, pp. 4751-4756.

[3] M. Khandaker and K.-K. Wong, "Robust secrecy beamforming with energy-harvesting eavesdroppers," IEEE Wireless Commun. Lett., vol. 4, no. 1, pp. 10-13, Feb 2015.

[4] T. A. Le, H. Nguyen, Q.-T. Vien, and M. Karamanoglu, "Secure information transmission and power transfer in cellular networks," IEEE Commun. Lett., vol. 19, no. 9, pp. 1532-1535, Sept 2015.

[5] L. Liu, R. Zhang, and K.-C. Chua, "Secrecy wireless information and power transfer with MISO beamforming," IEEE Trans. Signal Process., vol. 62, no. 7, pp. 1850-1863, Apr. 2014.

[6] Q. Shi, W. Xu, J. Wu, E. Song, and Y. Wang, "Secure beamforming for MIMO broadcasting with wireless information and power transfer," IEEE Trans. Wireless Commun., vol. 14, no. 5, pp. 2841-2853, May 2015.

[7] D. Ng, E. Lo, and R. Schober, "Robust beamforming for secure communication in systems with wireless information and power transfer," IEEE Trans. Wireless Commun., vol. 13, no. 8, pp. 4599-4615, Aug 2014.

[8] N. D. Sidiropoulos, T. N. Davidson, and Z.-Q. Luo, "Transmit beamforming for physical-layer multicasting," IEEE Trans. Signal Process., vol. 54, no. 6, pp. 2239-2251, Jun. 2006.

[9] A. H. Phan, H. D. Tuan, H. H. Kha, and D. T. Ngo, "Nonsmooth optimization for efficient beamforming in cognitive radio multicast transmission," IEEE Trans. Signal Process., vol. 60, no. 6, pp. 29412951, Jun. 2012.

[10] Y. Huang and D. P. Palomar, "Rank constrained separable semidefinite program with applications to optimal beamforming," IEEE Trans. Signal Process., vol. 2, no. 5, pp. 664-678, Feb 2010.

[11] X. Zhou, R. Zhang, and C. K. Ho, "Wireless information and power transfer: Architecture design and rate-energy tradeoff," IEEE Trans. Commun., vol. 61, no. 11, pp. 4757-4767, Nov. 2013.

[12] A. A. Nasir, D. T. Ngo, X. Zhou, R. A. Kennedy, and S. Durrani, "Joint resource optimization for multicell networks with wireless energy harvesting relays," IEEE Trans. Veh. Technol., 2015.

[13] H. D. Tuan, P. Apkarian, S. Hosoe, and H. Tuy, "D.C. optimization approach to robust control: Feasibility problems," Int. J. Contr, vol. 73 , no. 2, pp. 89-104, Feb. 2000.

[14] H. H. Kha, H. D. Tuan, H. H. Nguyen, and T. T. Pham, "Optimization of cooperative beamforming for SC-FDMA multi-user multi-relay networks by tractable D.C. programming," IEEE Trans. Signal Process., vol. 61, pp. 467-479, 2013.

[15] Q. Shi, L. Liu, W. Xu, and R. Zhang, "Joint transmit beamforming and receive power splitting for MISO SWIPT systems," IEEE Trans. Wireless Commun., vol. 13, no. 6, pp. 3269-3280, Jun. 2014. 